\title{
Structural gray matter differences in Problematic Usage of the Internet: a systematic review and meta-analysis
}

\author{
Jeremy E. Solly $\mathbb{D}^{1,2 \bowtie}$, Roxanne W. Hook ${ }^{2}$, Jon E. Grant ${ }^{3}$, Samuele Cortese $e^{4,5,6,7,8}$ and Samuel R. Chamberlain (iD) $2,5,9,10$ \\ (c) The Author(s) 2021
}

\begin{abstract}
Problematic Usage of the Internet (PUI) has been linked to diverse structural gray matter changes in individual data studies. However, no quantitative synthesis across studies has been conducted. We aimed to identify gray matter regions showing significant spatial convergence across neuroimaging studies in PUI. We searched PubMed and PsycINFO up to 10/03/2021 and included original, cross-sectional comparative studies that examined structural gray matter imaging in PUI versus control groups; reported a whole-brain analysis; and provided peak coordinates for gray matter differences. From a total of 624 potentially relevant studies, 15 (including 355 individuals with PUI and 363 controls) were included in a meta-analysis of voxel-based morphometry studies. Anatomical likelihood estimation (ALE) meta-analysis was performed using extracted coordinates and identified significant spatial convergence in the medial/superior frontal gyri, the left anterior cingulate cortex/cingulate gyrus, and the left middle frontal/precentral gyri. Datasets contributing to these findings all indicated reduced gray matter in cases compared to controls. In conclusion, voxel-based morphometric studies indicate replicable gray matter reductions in the dorsolateral prefrontal cortex and anterior cingulate cortex in PUI, regions implicated in reward processing and top-down inhibitory control. Further studies are required to understand the nature of gray matter differences across PUI behaviors, as well as the contribution of particular mental health disorders, and the influence of variation in study and sample characteristics.
\end{abstract}

Molecular Psychiatry (2022) 27:1000-1009; https://doi.org/10.1038/s41380-021-01315-7

\section{INTRODUCTION}

Although the Internet plays a useful role in many areas of life, a subset of Internet users develops Problematic Usage of the Internet (PUI), characterized by loss of control and adverse consequences, such as feelings of distress and functional impairment in daily life [1]. PUI is a broad term that refers to a range of excessive online activities, including general surfing, gaming, gambling, buying/shopping, pornography use, and social networking $[2,3]$. It has been linked to impaired functioning, high rates of psychiatric morbidity, and reduced quality of life [4-6]. Though operational definitions vary, meta-analytic evidence indicates a high prevalence of PUI and that rates appear to be escalating over time [7]. Thus, PUI is a growing public health concern.

Currently, gaming disorder and gambling disorder are included in the ICD-11 as disorders that may involve both online and offline behavior [8], while Internet Gaming Disorder (IGD) is included in the Diagnostic and Statistical Manual of Mental Disorders Version 5 (DSM-5) as a condition for further study [9]. Other examples of problematic Internet-related behaviors, such as social networking [10], are not currently included in classification systems. There is ongoing discussion regarding whether these behaviors are best classified using the concept of $\mathrm{PUI} /$ Internet addiction or as specific addictions to various Internet-related activities [11]. Despite the heterogeneity of PUI, a meta-analysis of cognitive deficits in PUI found that, irrespective of whether gaming was the predominant type of online behavior, PUI was characterized by pronounced impairment on cognitive domains which are known to be related to the fronto-striatal brain circuitry with small-medium effect sizes [12]. Further research is needed to understand the similarities and differences between different forms of PUI and between PUI and other mental disorders [3].

Whilst reproducible results have been reported in the neuropsychological literature, gray matter structural brain abnormalities have been variably associated with PUI, including in areas implicated in reward processing and top-down inhibitory control [13-18]. However, there are many discrepancies between published studies, which have found inconsistent abnormalities across diverse regions of the brain $[13,18]$. As neuroimaging literature grows, meta-analysis becomes an important tool to synthesize findings across studies [19]. Prior meta-analyses focusing on IGD using signed differential mapping identified lower gray matter volume in the anterior cingulate cortex (ACC), supplementary motor area (SMA), right putamen, right inferior frontal gyrus, and left dorsolateral prefrontal cortex (DLPFC)

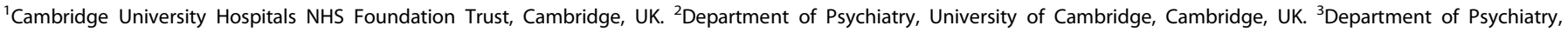

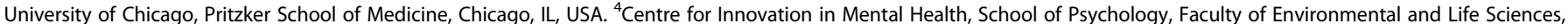

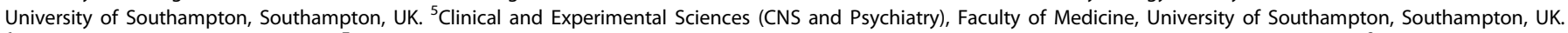

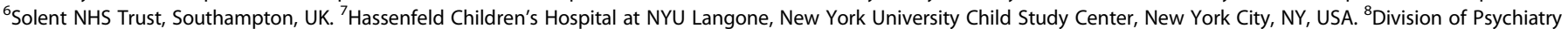

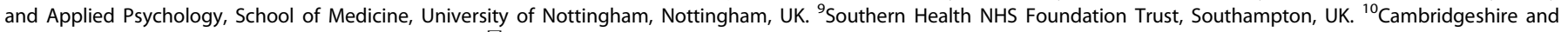
Peterborough NHS Foundation Trust, Cambridge, UK. ${ }^{\circledR}$ email: solly.j@doctors.org.uk
} 
$[20,21]$. However, meta-analysis of gray matter structural studies across the PUI literature has yet to be conducted.

Therefore, the aim of this study was to conduct the first systematic review and meta-analysis of gray matter structural imaging studies of PUI. We used anatomical likelihood estimation (ALE) methodology, which is a meta-analytic technique focusing on spatial convergence of foci rather than effect sizes [22-24]. Based on findings from neuropsychological studies in PUI, we hypothesized that PUI would be associated with abnormal gray matter in neural regions implicated in reward processing and topdown inhibitory control.

\section{MATERIALS AND METHODS}

The protocol for this study was pre-registered in the international prospective register of systematic reviews (PROSPERO 2020 CRD42020176234), which also indices any subsequent changes made to the protocol with a detailed rationale (Supplementary Methods 1 ). Study reporting followed guidelines for the reporting of neuroimaging meta-analysis [19] (Supplementary Table 1) and was compliant with Preferred Reporting Items for Systematic Reviews and Meta-Analyses (PRISMA) [25] (Supplementary Table 2) and Meta-analysis Of Observational Studies in Epidemiology (MOOSE) guidance [26] (Supplementary Table 3).

\section{Literature search}

PubMed and PsycINFO were searched from inception to 10/03/2021, using a search syntax modified from a previous meta-analysis of cognitive deficits in PUI [12] and adapted for the two databases: ("imaging" OR "MRI" OR "VBM" OR "voxel-based morphometry") AND ("internet use" OR "internet addiction" OR "smartphone use" OR "smartphone addiction" OR "gaming addiction" OR "internet gaming disorder" OR "PIU" OR "PUI"). This yielded a total of 546 hits in PubMed and 225 hits in PsycINFO, representing 613 unique records after de-duplication.

\section{Study selection}

Selection took place in two stages, screening and eligibility assessment (Fig. 1). In the screening stage, two researchers independently screened titles and abstracts. Studies were excluded if they were clearly out of scope, if they were not peer-reviewed publications, or not written in English. Our rationale for excluding non-English studies was that translation of manuscripts into English would have required access to specialist technical imaging expertize in a variety of languages, for which resourcing was not available in this study. All studies deemed relevant by either researcher proceeded to eligibility assessment. Reference lists of all studies proceeding to eligibility assessment and all identified relevant reviews/meta-analyses were hand-searched by one researcher and studies of possible relevance were identified and proceeded to eligibility assessment.

In the eligibility assessment stage, full texts were assessed independently by two researchers. Discrepancies were resolved by consensus discussion. Included studies: (1) were original, cross-sectional comparative studies that used structural gray matter imaging in a PUI population versus a control group, in which PUI was confirmed using a psychiatric interview or a validated questionnaire; (2) reported a whole-brain analysis (a requirement for the type of meta-analytic approach we used); and (3) reported peak coordinates for gray matter differences between the PUI and control groups. For studies that satisfied (1) but not (2) or (3), the authors were systematically contacted by email to inquire whether the required information was available. Studies reporting no significant peak coordinates in a whole-brain analysis were excluded.

Where there were concerns regarding possible overlapping samples between studies, authors were contacted for further information. In the absence of additional information, the study with the largest sample was included in the relevant meta-analysis.

\section{Data extraction and study quality}

Details regarding data extraction and extracted peak coordinates for each study are provided in the supplementary material (Supplementary Methods 3 and Supplementary Table 4). Peak coordinates were visualized with the BrainNet Viewer (http://www.nitrc.org/projects/bnv/) [27] (Fig. 2 and Supplementary Fig. 1).

Some studies reported multiple sets of "experiments" for the same group of subjects, where "experiment" refers to a single contrast producing a set of coordinates [28]. In these cases, results were combined in order that a maximum of two experiments per subject group (increases and decreases in gray matter, i.e., PUI > control and PUI < control) were included in the analysis, consistent with previous studies [28, 29].

To facilitate quality assessment, we designed a study quality checklist, which was adapted from checklists used in previous meta-analyses (Supplementary Methods 3). Relevant information was extracted from studies (Supplementary Table 5) and included in a qualitative literature synthesis.

\section{Statistical analysis}

We carried out coordinate-based meta-analysis using ALE, which is a metaanalytic technique focusing on spatial convergence of foci [22-24]. Using GingerALE Version 3.0.2 (http://www.brainmap.org/ale/), ALE was used to test for significant spatial convergence of foci between experiments, where the null hypothesis was random spatial association [29-31]. ALE first models foci as peaks of three-dimensional Gaussian distributions, to represent the spatial uncertainty of each reported coordinate, taking into account the number of subjects (experiments with fewer subjects have a wider Gaussian) [30]. A "modeled-activation" map for each experiment is created by merging these probability distributions, controlling for the effects of multiple foci being reported close together in a single experiment [29]. ALE scores are then calculated for each voxel by taking the union of the modeled-activation maps and tested against a null distribution with correction for multiple comparisons [31]. Cluster-level family-wise error correction was used, with a cluster-forming threshold on the voxel-level of $P<.001$ and a cluster-level threshold of $P<.05[19,32]$. As per the protocol and based on published simulations, at least 17 experiments were required to carry out an ALE meta-analysis, to control for the influence of any individual experiment [32].

Experiments included in each analysis are reported in the supplement (Supplementary Table 6). Significant clusters were visualized using Mango (rii.uthscsa.edu/mango) (Fig. 2) and interpreted directionally by inspecting those case-control studies directly contributing to the results.

Where significant clusters were identified, a leave-one-out jackknife sensitivity analysis was performed to assess the robustness of the results; i.e., the meta-analysis was repeated multiple times, leaving out one experiment each time. Results from each iteration were inspected to determine whether significant clusters were preserved (Supplementary Table 7) and, where clusters remained significant, whether their characteristics had altered (Supplementary Table 8).

\section{RESULTS}

From a total of 624 studies considered, 15 were identified for the main analysis focusing on VBM (including 355 individuals with PUI and 363 controls). Secondary analyses were also conducted, (a) focusing on studies examining IGD specifically (rather than PUI in general; 363 individuals with IGD and 373 controls); and (b) adding identified non-VBM studies to the VBM studies (455 individuals with PUI and 466 controls) (Fig. 1 and Supplementary Table 6). A full list of studies excluded at eligibility assessment, with reasons for exclusion, is provided in the supplementary material (Supplementary Methods 2).

Table 1 summarizes the characteristics of included studies [33-50] and Table 2 shows gray matter regions displaying significant differences between PUI and control groups for each study. Across the whole identified literature, six studies reported the use of a diagnostic interview to confirm PUI (Supplementary Table 5). Mood/anxiety disorders and attention-deficit/hyperactivity disorder (ADHD)/impulsivity were each assessed using validated questionnaires by 11 studies. None of the studies reported the use of a validated questionnaire to assess for impulse control/gambling comorbidities.

The VBM meta-analysis (19 experiments from 15 studies, including 73 foci) identified significant clusters mapping to the medial/superior frontal gyri; the left ACC/cingulate gyrus; and the left middle frontal/ precentral gyri (Fig. 2 and Table 3). The experiments contributing to these clusters all indicated reduced gray matter in PUI participants compared to controls (Table 3). The secondary analyses did not yield significant clusters (Table 3 and Supplementary Fig. 1). 


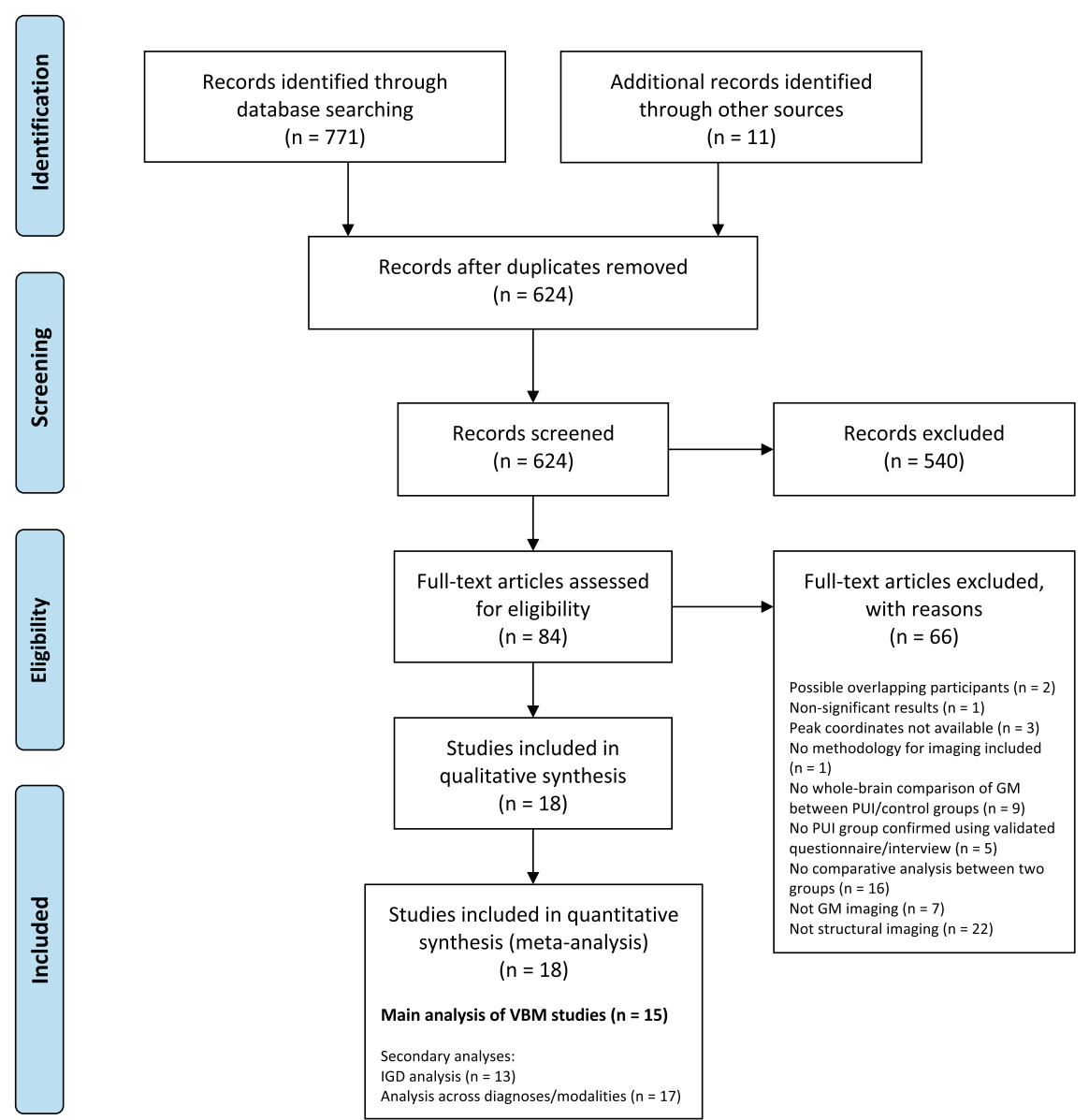

Fig. 1 Preferred reporting items for systematic reviews and meta-analyses (PRISMA) flow diagram. Diagram adapted from Moher et al. (2009) [25] using the template available from prisma-statement.org. The references of excluded full text articles are provided in the online supplement (Supplementary Methods 2). GM gray matter; IGD Internet gaming disorder; PUI Problematic Usage of the Internet; VBM voxelbased morphometry.

Leave-one-out jackknife analysis of the VBM meta-analysis demonstrated the following results. The two largest clusters mapping to the medial/superior frontal gyri and the left ACC/ cingulate gyrus were each replicated in 16 out of 19 iterations. The smaller cluster mapping to the left middle frontal/precentral gyri was replicated in 15 out of 19 iterations (Supplementary Table 7). Regions and extents of the jackknife findings are shown in Supplementary Table 8.

\section{DISCUSSION}

To our knowledge, this is the first ALE meta-analysis to investigate gray matter structural differences between individuals with PUI and controls. PUI was defined as encompassing problematic behaviors related to the Internet, irrespective of modality or type of activity. Focusing on voxel-based morphometry (VBM) studies, we found significantly reduced gray matter in PUI, versus controls, in the ACC, DLPFC, and SMA. These findings broadly confirmed our hypothesis given that these regions are heavily implicated, in other work, with reward processing and top-down inhibitory control [51, 52].

While previous non-ALE meta-analyses identified brain abnormalities in IGD [20, 21], we did not find, in our secondary analysis, significant abnormalities in IGD using ALE. We suspect this may relate to statistical power, as implicated regions in prior IGD work overlap with those herein found to be significantly abnormal in PUI. Also, our secondary analysis pooling all methodologies similarly did not identify significant abnormalities. This could reflect the loss of power arising from the inclusion of heterogeneous research methodologies. Indeed, there are discrepancies between gray matter volume and cortical thickness measures when applied to the same datasets and so they represent complementary, rather than interchangeable, measures $[53,54]$.

Meta-analytic confirmation of structural alterations in the ACC, DLPFC, and SMA in VBM studies provides insight into the possible neurobiology of PUI, even though structural abnormalities do not necessarily reflect functional abnormalities. The DLPFC and ACC are part of fronto-striatal circuitry that has been implicated in reward processing and inhibitory control; [51] hence, their dysfunction could contribute to elements of repetitive internetbased behavior. In the context of IGD, incongruent response errors in the Stroop task have been found to correlate with ACC structure, suggesting a role for the ACC in cognitive control [55], while ACC and DLPFC structure have also been correlated with impulsivity $[33,50]$. The SMA is involved in complex action and has been implicated in task-switching and stop-signal task performance [56]. Identification of structural differences in brain areas related to decision making and inhibitory control supports the Interaction of Person-Affect-Cognition-Execution model, proposed by Brand and colleagues, which suggests that reductions in executive functions and inhibitory control contribute to the development of Internet-use disorders [57].

It is important to consider possible methodological limitations relating to the literature that contributed to the analyses. 

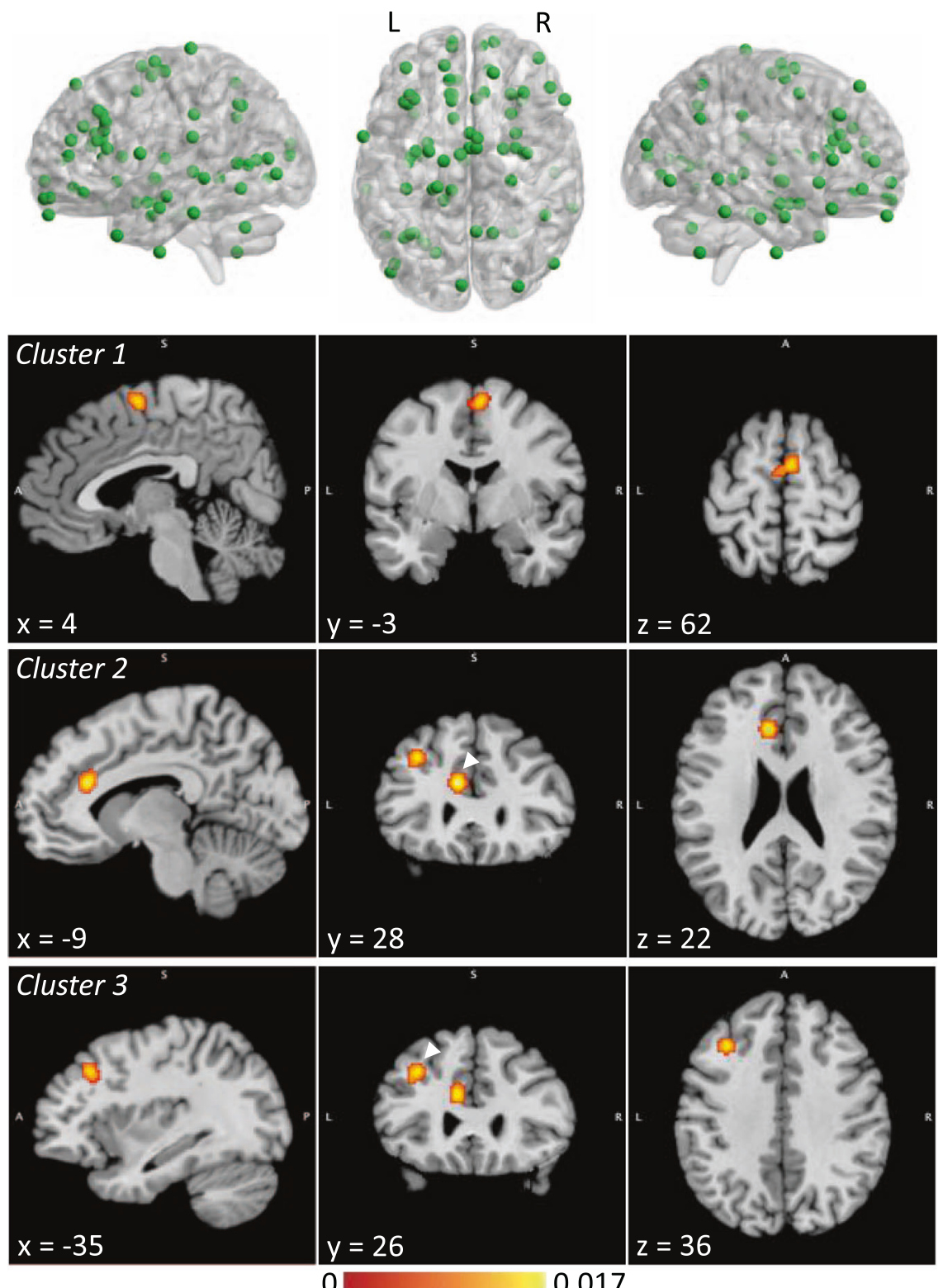

0

0.017

Fig. 2 Meta-analysis of voxel-based morphometry studies, showing coordinates included in the analysis and significant clusters. Clusters are shown at their weighted centers. The color bar shows ALE score. Where two clusters are seen in a single image, the relevant cluster is indicated with an arrowhead. ALE anatomical likelihood estimation; L left; R right.

Control group definitions varied between studies, with some studies recruiting participants reporting minimal Internet use and others recruiting regular Internet users (Table 1). There were also differences in diagnosis and comorbidity screening (Supplementary Table 5). A minority of studies (six out of eighteen) reported using a diagnostic interview to confirm PUI. Less than two-thirds reported the use of a formal screening tool assessing mood/ anxiety disorders and ADHD/impulsivity, which are known to be associated with PUI $[5,58]$. Finally, none of the included studies reported the use of a standardized tool for assessing impulse control/gambling such as the Minnesota Impulsive Disorders Interview [59]. Thus, further high-quality case-control neuroimaging studies are needed to fully elucidate the contribution of other underlying mental disorders to the neuroimaging findings associated with PUI.

In future, the adoption of consensus diagnostic criteria for PUI is expected to reduce heterogeneity. The use of clearly defined control groups, representing both participants with minimal Internet use and participants demonstrating high involvement but non-problematic use, will become important in identifying gray matter changes specific to PUI. This may involve changes to current widely-used criteria, as a Delphi study involving an international expert panel found that some published criteria for gaming disorder were not clinically relevant and some may not distinguish high but non-problematic involvement from problematic involvement in gaming [60]. In addition, the majority of studies included in the current meta-analysis investigated IGD, most participants were male, and most studies were conducted in China and in South Korea. In future, it will be important for studies to investigate a wider spectrum of PUI behaviors in a range of populations. By investigating a range of PUI behaviors and rigorously measuring the contribution of comorbidities, future studies will provide detailed information regarding the similarities and differences in gray matter structure between PUI subtypes 


\begin{tabular}{|c|c|c|c|c|c|c|c|c|c|}
\hline$\stackrel{\circ}{-}$ & 8 & $\bar{m}$ & 6 & $\stackrel{8}{0}$ & $\stackrel{8}{\circ}$ & $\stackrel{8}{0}$ & 8 & $\stackrel{8}{\circ}$ & $\infty$ \\
\hline $\begin{array}{l}\bar{a} \\
\dot{d} \\
\stackrel{\sim}{N}\end{array}$ & $\begin{array}{l}\overline{\bar{j}} \\
\text { à } \\
\dot{\nu}\end{array}$ & 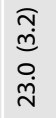 & $\begin{array}{l}\underset{\infty}{\infty} \\
\stackrel{\infty}{\infty} \\
\stackrel{\infty}{\infty} \\
\stackrel{\infty}{D}\end{array}$ & 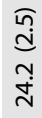 & 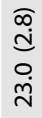 & 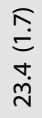 & $\begin{array}{l}\overline{1} \\
\mathfrak{N} \\
m \\
\end{array}$ & 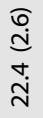 & 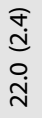 \\
\hline$\stackrel{\beth}{\sim}$ & $\stackrel{\infty}{\stackrel{\infty}{2}}$ & $\stackrel{\mathscr{N}}{ }$ & $\bar{\sim}$ & 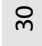 & $\stackrel{\text { m }}{ }$ & $\stackrel{m}{m}$ & $\stackrel{\circ}{\text { m }}$ & $\stackrel{\sim}{N}$ & $\bar{N}$ \\
\hline 8 & 8 & $\tilde{m}$ & $\mathcal{H}_{0}$ & 음 & $\stackrel{8}{\circ}$ & 음 & 므 & 음 & $\infty$ \\
\hline
\end{tabular}

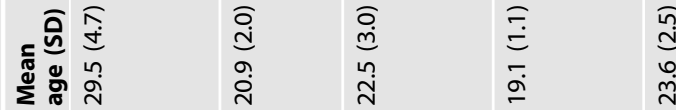

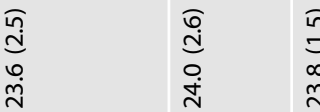

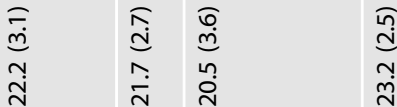

న $\geq$ ก

กิ

$\stackrel{n}{\sim}$

$\bar{m} \quad$ \&

$\stackrel{n}{m}$

กั $\stackrel{\infty}{=}$

$\stackrel{\circ}{\sim}$
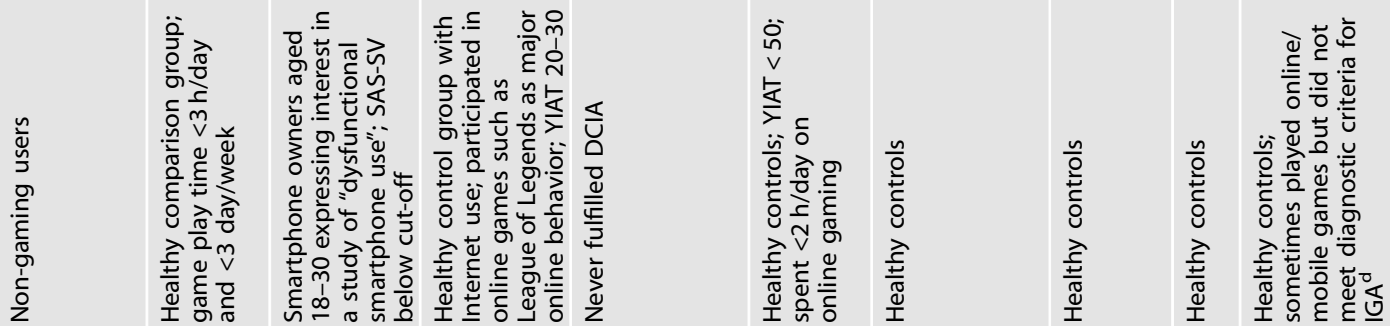

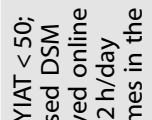

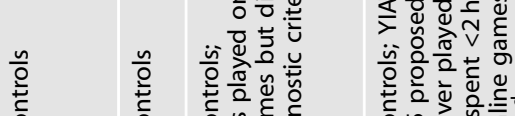

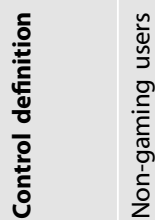

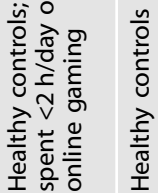

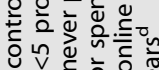

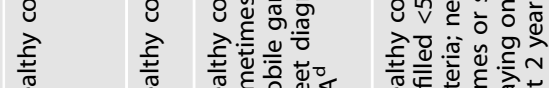

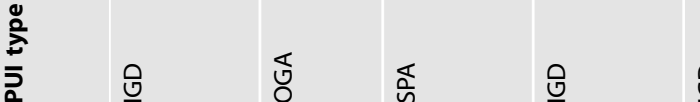

요

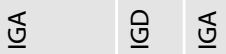

$\underline{\mathrm{o}}$

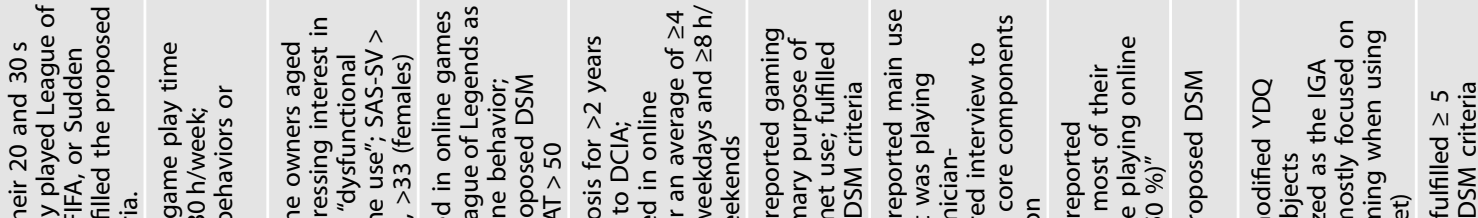

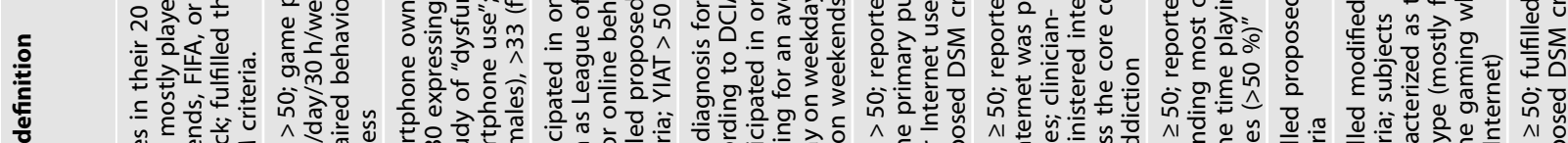

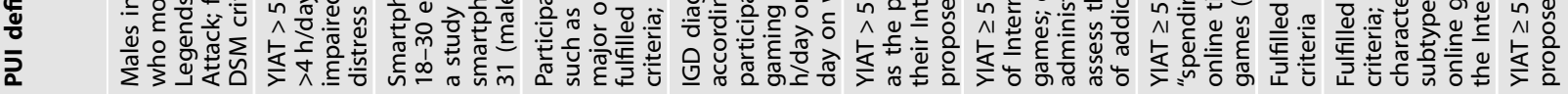

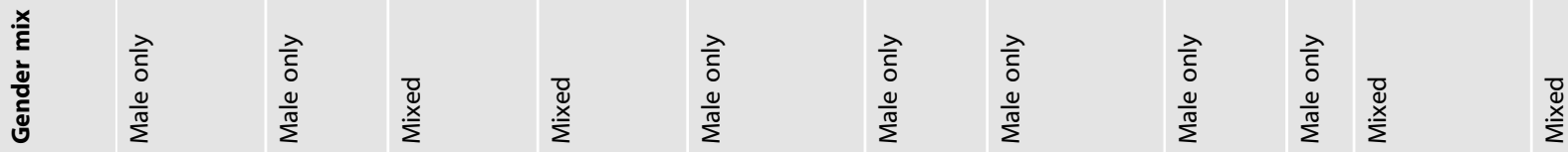

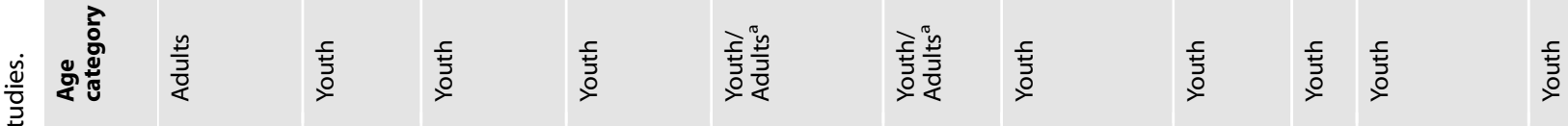

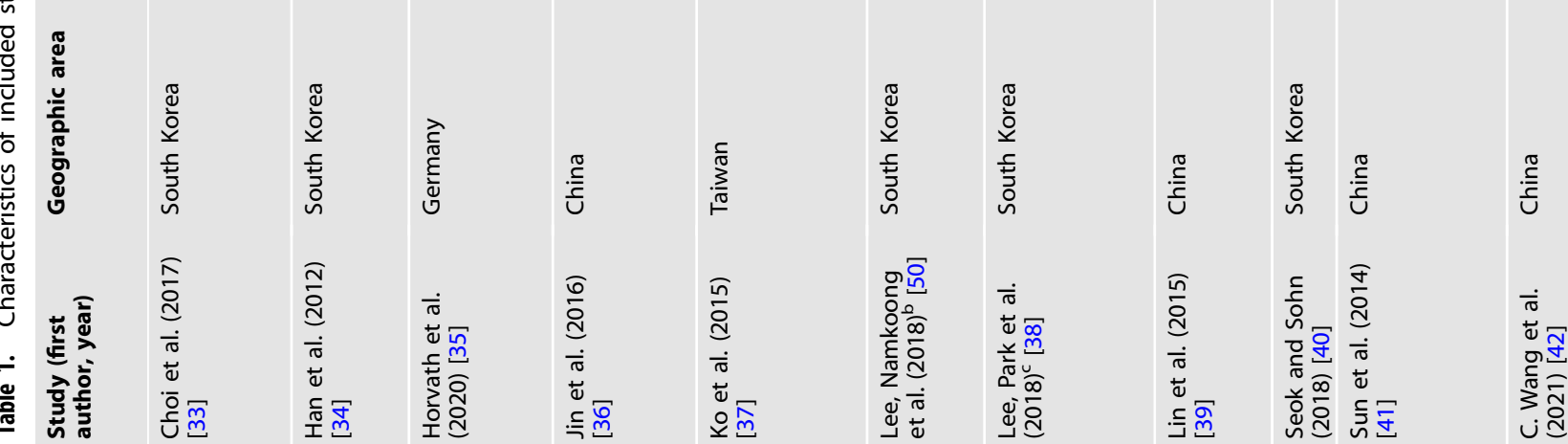




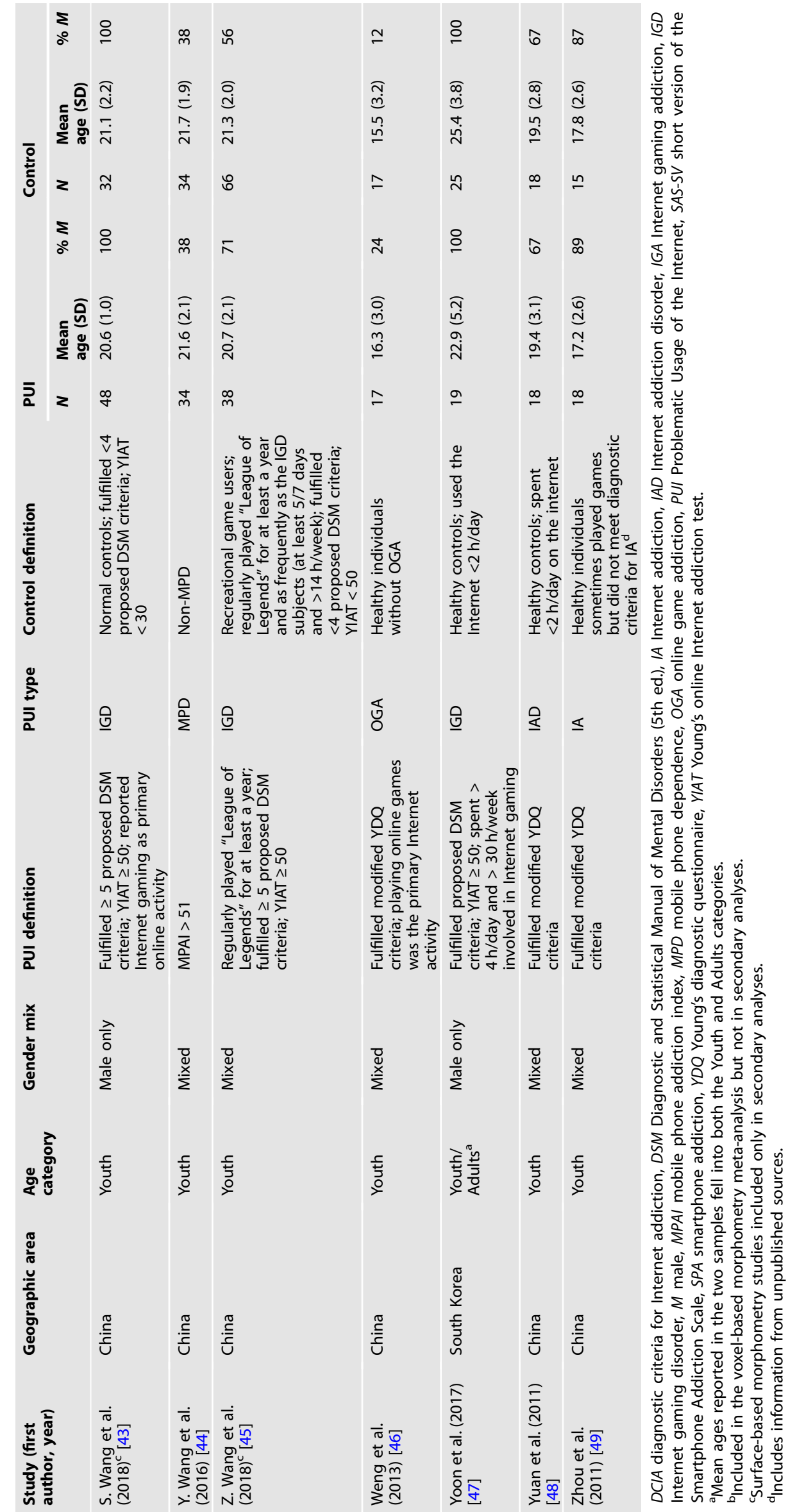


Table 2. Gray matter regions with significant differences between PUI and control groups in included studies.

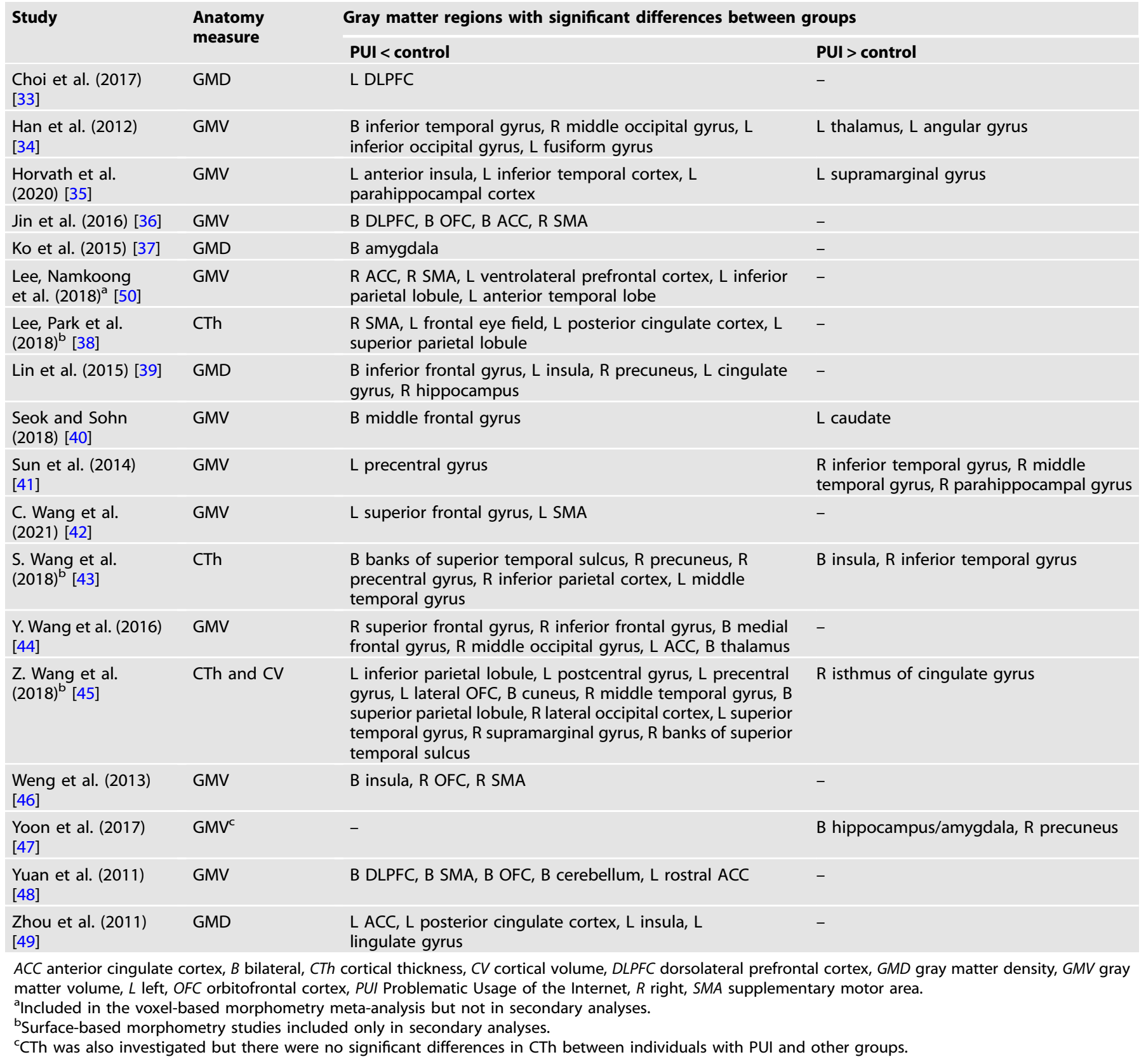

and how these relate to brain structural differences identified in other mental disorders.

\section{Strengths and limitations}

The strengths of this study include the use of an inclusive search strategy to systematically identify relevant studies, coupled with a systematic process to gather unpublished information/data from study authors, and exclusion of potentially duplicated datasets. An important limitation of the current study is that it was not possible to assess the contributions of specific study and sample characteristics to clusters identified in ALE analysis, as each cluster result was primarily driven by a relatively small number of studies. In addition, it was not possible to carry out subgroup metaanalyses to assess, formally, particular directions of gray matter change (i.e., increases and decreases) within ALE itself due to a small number of eligible experiments. Therefore, the current study interpreted directionality by inspecting findings from case-control studies contributing significantly to a given ALE cluster. For resource availability reasons, another limitation is that the study did not include non-English publications. Finally, as our analysis used ALE and the null hypothesis was a lack of spatial convergence across the whole brain, it was not possible to include studies reporting non-significant results or studies using only region-of-interest analysis [19]. Only one study was excluded due to a lack of significant results (Supplementary Methods 2). There have been a number of studies assessing gray matter changes in particular regions-of-interest in PUI, including the striatum $[33,61-68]$, frontal cortex $[38,61,69-73]$, cingulate cortex $[38,63,66,67,71,73]$, amygdala $[61,63,66,67]$, insula $[73,74]$ and temporal cortex [73]. 


\section{CONCLUSIONS}

This meta-analysis of VBM studies confirmed reduced gray matter of the SMA, left ACC, and left DLPFC in PUI. Further research is needed to understand the nature of gray matter differences across different PUI behaviors (such as whether they constitute vulnerability markers or stem from PUI itself) and elucidate the contribution of underlying mental health diagnoses, as well as the influence of variation in study and participant characteristics on such findings.

\section{REFERENCES}

1. Shapira NA, Lessig MC, Goldsmith TD, Szabo ST, Lazoritz M, Gold MS, et al. Problematic internet use: proposed classification and diagnostic criteria. Depress Anxiety. 2003;17:207-16.

2. Ioannidis K, Treder MS, Chamberlain SR, Kiraly F, Redden SA, Stein DJ, et al. Problematic internet use as an age-related multifaceted problem: evidence from a two-site survey. Addict Behav. 2018;81:157-66.

3. Fineberg NA, Demetrovics Z, Stein DJ, loannidis K, Potenza MN, Grünblatt E, et al. Manifesto for a European research network into problematic usage of the Internet. Eur Neuropsychopharmacol. 2018;28:1232-46.

4. Derbyshire KL, Lust KA, Schreiber LRN, Odlaug BL, Christenson GA, Golden DJ, et al. Problematic Internet use and associated risks in a college sample. Compr Psychiatry. 2013;54:415-22.

5. Ho RC, Zhang MWB, Tsang TY, Toh AH, Pan F, Lu Y, et al. The association between internet addiction and psychiatric co-morbidity: a meta-analysis. BMC Psychiatry. 2014;14:183.

6. Chamberlain SR, loannidis K, Grant JE. The impact of comorbid impulsive/compulsive disorders in problematic Internet use. J Behav Addict. 2018;7:269-75.

7. Pan YC, Chiu YC, Lin YH. Systematic review and meta-analysis of epidemiology of internet addiction. Neurosci Biobehav Rev. 2020;118:612-22.

8. World Health Organization. International classification of diseases for mortality and morbidity statistics (11th revision). https://icd.who.int/.

9. American Psychiatric Association. Diagnostic and statistical manual of mental disorders. 5th ed. Arlington, VA: American Psychiatric Association; 2013.

10. Kuss DJ, Griffiths MD. Online social networking and addiction-a review of the psychological literature. Int J Environ Res Public Health. 2011;8:3528-52.

11. Starcevic V, Billieux J. Does the construct of Internet addiction reflect a single entity or a spectrum of disorders? Clin Neuropsychiatry. 2017;14:5-10.

12. Ioannidis $K$, Hook R, Goudriaan AE, Vlies S, Fineberg NA, Grant JE, et al. Cognitive deficits in problematic internet use: meta-analysis of 40 studies. $\mathrm{Br} J$ Psychiatry. 2019;215:639-46.

13. Park $B, H a n D H$, Roh $S$. Neurobiological findings related to internet use disorders. Psychiatry Clin Neurosci. 2017;71:467-78.

14. Weinstein A, Lejoyeux M. New developments on the neurobiological and pharmaco-genetic mechanisms underlying internet and videogame addiction. Am J Addict. 2015;24:117-25.

15. Weinstein A, Lejoyeux M. Neurobiological mechanisms underlying internet and gaming disorder (IGD). Dialogues Clin Neurosci. 2020;22:113-26.

16. Weinstein AM. An update overview on brain imaging studies of internet gaming disorder. Front Psychiatry. 2017;8:185.

17. Weinstein A, Livny A, Weizman A. New developments in brain research of internet and gaming disorder. Neurosci Biobehav Rev. 2017;75:314-30.

18. Kuss DJ, Pontes HM, Griffiths MD. Neurobiological correlates in internet gaming disorder: a systematic literature review. Front Psychiatry. 2018;9:166.

19. Müller Vl, Cieslik EC, Laird AR, Fox PT, Radua J, Mataix-Cols D, et al. Ten simple rules for neuroimaging meta-analysis. Neurosci Biobehav Rev. 2018;84:151-61.

20. Yao YW, Liu L, Ma SS, Shi XH, Zhou N, Zhang JT, et al. Functional and structural neural alterations in Internet gaming disorder: a systematic review and metaanalysis. Neurosci Biobehav Rev. 2017;83:313-24.

21. Qin K, Zhang F, Chen T, Li L, Li W, Suo X, et al. Shared gray matter alterations in individuals with diverse behavioral addictions: a voxel-wise meta-analysis. J Behav Addict. 2020;9:44-57.

22. Acar F, Seurinck R, Eickhoff SB, Moerkerke B. Assessing robustness against potential publication bias in Activation Likelihood Estimation (ALE) meta-analyses for fMRI. PLoS One. 2018;13:e0208177.

23. Radua J, Rubia K, Canales-Rodríguez EJ, Pomarol-Clotet E, Fusar-Poli P, MataixCols D. Anisotropic kernels for coordinate-based meta-analyses of neuroimaging studies. Front Psychiatry. 2014;5:13.

24. Ellison-Wright I, Glahn DC, Laird AR, Thelen SM, Bullmore E. The anatomy of firstepisode and chronic schizophrenia: an anatomical likelihood estimation metaanalysis. Am J Psychiatry. 2008;165:1015-23.

25. Moher D, Liberati A, Tetzlaff J, Altman DG. Preferred reporting items for systematic reviews and meta-analyses: the PRISMA statement. PLoS Med. 2009;6:e1000097. 
26. Stroup DF, Berlin JA, Morton SC, Olkin I, Williamson GD, Rennie D, et al. Metaanalysis of observational studies in epidemiology: a proposal for reporting. JAMA 2000;283:2008-12.

27. Xia M, Wang J, He Y. BrainNet viewer: a network visualization tool for human brain connectomics. PLoS One. 2013;8:e68910.

28. Samea F, Soluki S, Nejati V, Zarei M, Cortese S, Eickhoff SB, et al. Brain alterations in children/adolescents with $A D H D$ revisited: a neuroimaging metaanalysis of 96 structural and functional studies. Neurosci Biobehav Rev. 2019;100:1-8.

29. Turkeltaub PE, Eickhoff SB, Laird AR, Fox $M$, Wiener M, Fox P. Minimizing withinexperiment and within-group effects in activation likelihood estimation metaanalyses. Hum Brain Mapp. 2012;33:1-13.

30. Eickhoff SB, Laird AR, Grefkes C, Wang LE, Zilles K, Fox PT. Coordinate-based activation likelihood estimation meta-analysis of neuroimaging data: a randomeffects approach based on empirical estimates of spatial uncertainty. Hum Brain Mapp. 2009;30:2907-26.

31. Eickhoff SB, Bzdok D, Laird AR, Kurth F, Fox PT. Activation likelihood estimation meta-analysis revisited. Neuroimage 2012;59:2349-61.

32. Eickhoff SB, Nichols TE, Laird AR, Hoffstaedter F, Amunts K, Fox PT, et al. Behavior, sensitivity, and power of activation likelihood estimation characterized by massive empirical simulation. Neuroimage 2016;137:70-85.

33. Choi J, Cho H, Kim JY, Jung DJ, Ahn KJ, Kang HB, et al. Structural alterations in the prefrontal cortex mediate the relationship between Internet gaming disorder and depressed mood. Sci Rep. 2017;7:1245.

34. Han DH, Lyoo IK, Renshaw PF. Differential regional gray matter volumes in patients with on-line game addiction and professional gamers. J Psychiatr Res. 2012;46:507-15.

35. Horvath J, Mundinger C, Schmitgen MM, Wolf ND, Sambataro F, Hirjak D, et al. Structural and functional correlates of smartphone addiction. Addict Behav. 2020;105:106334.

36. Jin C, Zhang T, Cai C, Bi Y, Li Y, Yu D, et al. Abnormal prefrontal cortex resting state functional connectivity and severity of internet gaming disorder. Brain Imaging Behav. 2016;10:719-29.

37. Ko CH, Hsieh TJ, Wang PW, Lin WC, Yen CF, Chen CS, et al. Altered gray matter density and disrupted functional connectivity of the amygdala in adults with Internet gaming disorder. Prog Neuropsychopharmacol Biol Psychiatry. 2015;57:185-92.

38. Lee D, Park J, Namkoong K, Kim IY, Jung YC. Gray matter differences in the anterior cingulate and orbitofrontal cortex of young adults with Internet gaming disorder: Surface-based morphometry. J Behav Addict. 2018;7:21-30.

39. Lin X, Dong G, Wang Q, Du X. Abnormal gray matter and white matter volume in 'Internet gaming addicts'. Addict Behav. 2015;40:137-43.

40. Seok JW, Sohn JH. Altered gray matter volume and resting-state connectivity in individuals with internet gaming disorder: a voxel-based morphometry and resting-state functional magnetic resonance imaging study. Front Psychiatry. 2018;9:77.

41. Sun $Y$, Sun J, Zhou $Y$, Ding W, Chen $X$, Zhuang Z, et al. Assessment of in vivo microstructure alterations in gray matter using DKI in internet gaming addiction. Behav Brain Funct. 2014;10:37.

42. Wang C, Zhang Z, Che L, Wu Y, Qian H, Guo X. The gray matter volume in superior frontal gyrus mediates the impact of reflection on emotion in Internet gaming addicts. Psychiatry Res Neuroimaging. 2021;310:111269.

43. Wang S, Liu J, Tian L, Chen L, Wang J, Tang Q, et al. Increased insular cortical thickness associated with symptom severity in male youths with internet gaming disorder: A surface-based morphometric study. Front Psychiatry. 2018;9:99.

44. Wang $Y$, Zou Z, Song $H, X u X$, Wang $H$, D'Oleire Uquillas $F$, et al. Altered gray matter volume and white matter integrity in college students with mobile phone dependence. Front Psychol. 2016;7:597.

45. Wang Z, Wu L, Yuan K, Hu Y, Zheng H, Du X, et al. Cortical thickness and volume abnormalities in Internet gaming disorder: evidence from comparison of recreational Internet game users. Eur J Neurosci. 2018;48:1654-66.

46. Weng $C B$, Qian RB, Fu XM, Lin B, Han XP, Niu CS, et al. Gray matter and white matter abnormalities in online game addiction. Eur J Radio. 2013;82:1308-12.

47. Yoon EJ, Choi JS, Kim H, Sohn BK, Jung HY, Lee JY, et al. Altered hippocampal volume and functional connectivity in males with Internet gaming disorder comparing to those with alcohol use disorder. Sci Rep. 2017:7:5744.

48. Yuan K, Qin W, Wang G, Zeng F, Zhao L, Yang X, et al. Microstructure abnormalities in adolescents with internet addiction disorder. PLoS One. 2011; 6:e20708.

49. Zhou Y, Lin FC, Du YS, Qin LD, Zhao ZM, Xu JR, et al. Gray matter abnormalities in internet addiction: a voxel-based morphometry study. Eur J Radio. 2011;79:92-95.

50. Lee $D$, Namkoong K, Lee J, Jung YC. Abnormal gray matter volume and impulsivity in young adults with Internet gaming disorder. Addict Biol. 2018;23:1160-7.

51. Volkow ND, Wang GJ, Tomasi D, Baler RD. Unbalanced neuronal circuits in addiction. Curr Opin Neurobiol. 2013;23:639-48.
52. Feil J, Sheppard D, Fitzgerald PB, Yücel M, Lubman DI, Bradshaw JL. Addiction, compulsive drug seeking, and the role of frontostriatal mechanisms in regulating inhibitory control. Neurosci Biobehav Rev. 2010;35:248-75.

53. Kong L, Herold CJ, Zöllner F, Salat DH, Lässer MM, Schmid LA, et al. Comparison of grey matter volume and thickness for analysing cortical changes in chronic schizophrenia: A matter of surface area, grey/white matter intensity contrast, and curvature. Psychiatry Res-Neuroimaging. 2015;231:176-83.

54. Hutton C, Draganski B, Ashburner J, Weiskopf N. A comparison between voxelbased cortical thickness and voxel-based morphometry in normal aging. Neuroimage 2009;48:371-80.

55. Wang H, Jin C, Yuan K, Shakir TM, Mao C, Niu X, et al. The alteration of gray matter volume and cognitive control in adolescents with internet gaming disorder. Front Behav Neurosci. 2015;9:64.

56. Nachev $P$, Kennard $C$, Husain M. Functional role of the supplementary and presupplementary motor areas. Nat Rev Neurosci. 2008;9:856-69.

57. Brand M, Young KS, Laier C, Wölfling K, Potenza MN. Integrating psychological and neurobiological considerations regarding the development and maintenance of specific Internet-use disorders: an Interaction of Person-AffectCognition-Execution (I-PACE) model. Neurosci Biobehav Rev. 2016;71:252-66.

58. Pettorruso M, Valle S, Cavic E, Martinotti G, di Giannantonio M, Grant JE. Problematic Internet use (PIU), personality profiles and emotion dysregulation in a cohort of young adults: trajectories from risky behaviors to addiction. Psychiatry Res. 2020;289:113036.

59. Grant JE, Levine L, Kim D, Potenza MN. Impulse control disorders in adult psychiatric inpatients. Am J Psychiatry. 2005;162:2184-8.

60. Castro-Calvo J, King DL, Stein DJ, Brand M, Carmi L, Chamberlain SR, et al. Expert appraisal of criteria for assessing gaming disorder: an international Delphi study. Addiction. 2021:116:2463-75.

61. Altbäcker A, Plózer E, Darnai G, Perlaki G, Horváth R, Orsi G, et al. Problematic internet use is associated with structural alterations in the brain reward system in females. Brain Imaging Behav. 2016;10:953-9.

62. Cai C, Yuan K, Yin J, Feng D, Bi Y, Li Y, et al. Striatum morphometry is associated with cognitive control deficits and symptom severity in internet gaming disorder. Brain Imaging Behav. 2016;10:12-20.

63. He Q, Turel O, Bechara A. Brain anatomy alterations associated with Social Networking Site (SNS) addiction. Sci Rep. 2017;7:45064.

64. Liu L, Yip SW, Zhang JT, Wang L, Shen ZJ, Liu B, et al. Activation of the ventral and dorsal striatum during cue reactivity in Internet gaming disorder. Addict Biol. 2017;22:791-801.

65. Montag C, Markowetz A, Blaszkiewicz K, Andone I, Lachmann B, Sariyska R, et al. Facebook usage on smartphones and gray matter volume of the nucleus accumbens. Behav Brain Res. 2017:329:221-8.

66. Montag C, Zhao Z, Sindermann C, Xu L, Fu M, Li J, et al. Internet Communication Disorder and the structure of the human brain: initial insights on WeChat addiction. Sci Rep. 2018;8:2155

67. Schmidt C, Morris LS, Kvamme TL, Hall P, Birchard T, Voon V. Compulsive sexual behavior: prefrontal and limbic volume and interactions. Hum Brain Mapp. 2017;38:1182-90.

68. Yuan K, Yu D, Cai C, Feng D, Li Y, Bi Y, et al. Frontostriatal circuits, resting state functional connectivity and cognitive control in internet gaming disorder. Addict Biol. 2017:22:813-22.

69. Hong SB, Kim JW, Choi EJ, Kim HH, Suh JE, Kim CD, et al. Reduced orbitofrontal cortical thickness in male adolescents with internet addiction. Behav Brain Funct. 2013;9:11.

70. Inhóf $\mathrm{O}$, Zsidó AN, Perlaki G, Orsi G, Lábadi B, Kovács $\mathrm{N}$, et al. Internet addiction associated with right pars opercularis in females. J Behav Addict. 2019;8:162-8.

71. Lee $D$, Namkoong $K$, Lee J, Lee $B O$, Jung YC. Lateral orbitofrontal gray matter abnormalities in subjects with problematic smartphone use. J Behav Addict. 2019;8:404-11.

72. Zhou F, Montag C, Sariyska R, Lachmann B, Reuter M, Weber B, et al. Orbitofrontal gray matter deficits as marker of internet gaming disorder: converging evidence from a cross-sectional and prospective longitudinal design. Addict Biol. 2019;24:100-9.

73. Zsidó AN, Darnai G, Inhóf O, Perlaki G, Orsi G, Nagy SA, et al. Differentiation between young adult Internet addicts, smokers, and healthy controls by the interaction between impulsivity and temporal lobe thickness. J Behav Addict. 2019;8:35-47.

74. Turel $\mathrm{O}, \mathrm{He} \mathrm{Q}$, Brevers $\mathrm{D}$, Bechara A. Delay discounting mediates the association between posterior insular cortex volume and social media addiction symptoms. Cogn Affect Behav Neurosci. 2018;18:694-704.

\section{ACKNOWLEDGEMENTS}

We would like to thank all authors of included studies who responded to requests for additional information. Dr. Solly's role in this paper was funded by the East Anglian 
Foundation Program. Dr. Chamberlain's role in this paper was funded by a Clinical Fellowship from the Wellcome Trust (reference 110049/Z/15/Z \& 110049/Z/15/A). This article/publication is based upon work from COST Action CA16207 "European Network for Problematic Usage of the Internet", supported by COST (European Cooperation in Science and Technology). www.cost.eu.

\section{AUTHOR CONTRIBUTIONS}

JES, RWH, JEG, SC, and SRC contributed to the study conceptualization and design. JES and RWH completed the literature search and data extraction, with supervision by SC and SRC. JES and SRC assessed study quality. JES carried out data analysis and wrote the manuscript draft, with supervision by SC and SRC. JES, RWH, JEG, SC, and SRC contributed to paper revision and read and approved the final paper.

\section{COMPETING INTERESTS}

Dr. Grant has received research grants from Biohaven, Promentis, and Otsuka Pharmaceuticals. Dr. Grant receives yearly compensation from Springer Publishing for acting as Editor-in-Chief of the Journal of Gambling Studies and has received royalties from Oxford University Press, American Psychiatric Publishing, Inc., Norton Press, and McGraw Hill. Dr. Chamberlain previously consulted (past 3 years) for Promentis on work unrelated to the content of this paper. He also receives an honorarium from Elsevier from editorial work at Comprehensive Psychiatry, and at Neuroscience \& Biobehavioral Reviews. Dr. Cortese declares honoraria and reimbursement for travel and accommodation expenses for lectures from the following non-profit associations: Association for Child and Adolescent Mental Health (ACAMH), Canadian ADHD Resource Alliance (CADDRA), British Association of Psychopharmacology (BAP), and from Healthcare Convention for educational activity on ADHD. Dr. Solly and Ms. Hook declare no potential competing interests.

\section{ADDITIONAL INFORMATION}

Supplementary information The online version contains supplementary material available at https://doi.org/10.1038/s41380-021-01315-7.

Correspondence and requests for materials should be addressed to Jeremy E. Solly.

Reprints and permission information is available at http://www.nature.com/ reprints

Publisher's note Springer Nature remains neutral with regard to jurisdictional claims in published maps and institutional affiliations.

Open Access This article is licensed under a Creative Commons Attribution 4.0 International License, which permits use, sharing, adaptation, distribution and reproduction in any medium or format, as long as you give appropriate credit to the original author(s) and the source, provide a link to the Creative Commons license, and indicate if changes were made. The images or other third party material in this article are included in the article's Creative Commons license, unless indicated otherwise in a credit line to the material. If material is not included in the article's Creative Commons license and your intended use is not permitted by statutory regulation or exceeds the permitted use, you will need to obtain permission directly from the copyright holder. To view a copy of this license, visit http://creativecommons. org/licenses/by/4.0/.

C The Author(s) 2021 